\title{
RECENT DEVELOPMENTS IN HEAVY FLAVOUR PRODUCTION
}

\author{
G. KRAMER \\ Universität Hamburg - II. Institut für Theoretische Physik \\ Luruper Chaussee 149 - 22761 Hamburg - Germany
}

\begin{abstract}
We review one-particle inclusive production of heavy-flavoured hadrons in a framework which resums the large collinear logarithms through the evolution of the FFs and PDFs and retains the full dependence on the heavy-quark mass without additional theoretical assumptions. We focus on presenting results for the inclusive cross section for the production of charmed mesons in $p \bar{p}$ collisions and the comparison with CDF data from the Tevatron as well as on inclusive $B$-meson production and comparison with recent CDF data. The third topic is the production of $D^{\star}$ mesons in photoproduction and comparison with recent $\mathrm{H} 1$ data from HERA.
\end{abstract}

\section{Introduction}

One-particle inclusive production processes provide extensive tests of perturbative quantum chromodynamics (pQCD). In contrast to fully inclusive processes, it is possible to study distributions in the momentum of the final-state particle and to apply kinematical cuts close to the experimental situation. On the other hand, contrary to even more exclusive cases, QCD factorisation theorems [2,3] still hold stating that this class of observables can be computed as convolutions of universal parton distribution functions (PDFs) and fragmentation functions (FFs) with perturbatively calculable hard scattering cross sections. As is well-known, it is due to the factorisation property that the parton model of QCD has predictive power. Hence, tests of the universality of the PDFs and FFs are of crucial importance for validating this QCD framework. At the same time, lowest-order expressions for the hard scattering cross sections are often not sufficient for meaningful tests and the use of higher order computations is needed.

The perturbative analysis is becoming more involved and interesting if the observed final state hadron contains a heavy (charm or bottom) quark. In this case, the heavy-quark mass $m$ enters as an additional scale. Clearly, the conventional massless formalism, also known as zero-mass variable-flavour-number scheme (ZM-VFNS), can also be applied to this case, provided the hard scale $Q$ of the process is much bigger than the heavy-quark mass so that terms $m / Q$ are negligible. However, at present collider energies, most of the experimental data lie in the kinematic region $Q \gtrsim m$ and it is necessary to take the power-like mass terms into account in a consistent framework.

The conventional calculational scheme is the so-called massive scheme or fixed-flavournumber scheme (FFNS) [4], in which the number of active flavours in the initial state is limited to $n_{f}=3\left(n_{f}=4\right)$ in the case of massive charm (bottom) production, and the $c$ (b) quark appears only in the final state. In this case, the $c(b)$ quark is always treated as a heavy particle, not as a parton. The actual mass parameter $m$ is explicitly taken into account along with $p_{T}$. In this scheme, $m$ acts as a cutoff for the initial- and final sate collinear singularities and sets the scale for the perturbative calculations. A factorisation of these would-be initial- and final state collinear singularities is not necessary, neither is the introduction of a FF for the transition $b \rightarrow B$. However at NLO, terms proportional to 
$\alpha_{s} \ln \left(p_{T}^{2} / m^{2}\right)$, where $\alpha_{s}$ is the strong coupling constant, arise from collinear gluon emissions by $c(b)$ quarks or from branchings of gluons into collinear $c \bar{c}(b \bar{b})$ pairs. These terms are of order $O(1)$ for large $p_{T}$ and spoil the convergence of the perturbation series. The FFNS with $n_{f}=3\left(n_{f}=4\right)$ should be limited to a rather limited range of $p_{T}$, from $p_{T}=0$ to $p_{T} \gtrsim m$. The advantage of this scheme, however, is that the $m^{2} / p_{T}^{2}$ power terms are fully taken into account.

The ZM-VFNS and FFNS are valid in complementary regions of $p_{T}$, and it is desirable to combine them in a unified approach that incorporates the advantages of both schemes, i.e. to resum the large logarithms, retain the full finite- $m$ effects, and preserve the universality of the FFs. An earlier approach to implement such an interpolation is the so-called fixedorder-next-to-leading logarithm (FONLL) scheme, in which the conventional cross section in the FFNS is linearly combined wit a suitably modified cross section in the ZM-VFNS with perturbative FFs, using a $p_{T}$-dependent weight function [5]. Then the FONLL cross section is convoluted with a non-pertubative $\mathrm{FF}$ for the $b \rightarrow B$ transition.

The subject of this review is the theoretical description of one-particle inclusive production of heavy-flavoured hadrons $X_{h}=D, B, \Lambda_{c}, \ldots$ in a massive variable-flavour-number scheme (GM-VFNS). In such a scheme the large collinear logarithms of the heavy-quark mass $\ln \mu / m$ are subtracted from the hard scattering cross sections and resummed through the evolution of the FFs and PDFs. At the same time, finite non-logarithmic mass terms $m / Q$ are retained in the hard part and fully taken into account.

In order to test the pQCD formalism, in particular the universality of the FFs, it is important to provide a description of all relevant processes in a coherent framework. Therefore, it is important to work out the GM-VFNS at next-to-leading order (NLO) of QCD for all the relevant processes. Previously, the GM-VFNS has been applied to the following processes: $\gamma+\gamma \rightarrow D^{\star+}+X$ (direct part) [6], $\gamma+\gamma \rightarrow D^{\star+}+X$ (single resolved part) [7], $\gamma+p \rightarrow D^{\star+}+X$ (direct part) [8], $p+\bar{p} \rightarrow\left(D^{0}, D^{\star+}, D^{+}, D_{s}^{+}\right)+X[9,10,11]$, where the latter results for hadron-hadron collisions also constitute the resolved contribution to the photoproduction process $\gamma+p \rightarrow X_{h}+X$.

In this contribution, I will review the progress achieved in describing the production of heavy-flavoured hadrons $X_{h}$ in hadron-hadron and photon-proton collisions in the GMVFN scheme as it has been worked out recently. The main focus will be on the comparison with experimental data from CDF at the Tevatron for $p+\bar{p} \rightarrow\left(D^{0}, D^{*+}, D^{+}, D_{s}^{+}\right)+X$ and $p+\bar{p} \rightarrow B^{+}+X$ and from H1 at HERA for $\gamma+p \rightarrow D^{*+}+X$.

\section{Theoretical Framework}

\subsection{GM-VFNS}

The differential cross sections for inclusive heavy-flavoured hadron production can be computed in the GM-VFNS according to the familiar factorisation formulae, however, with heavy-quark mass terms included in the hard scattering cross sections [12]. Generically, the physical cross sections are expressed as convolutions of PDFs for the incoming hadron(s), hard scattering cross sections, and FFs for the fragmentation of the outgoing partons into the observed hadron. All possible partonic subprocesses are taken into account. The massive hard scattering cross sections are constructed in a way that in the limit $m \rightarrow 0$ the conventional ZM-VFNS is recovered. A more detailed discussion of the GM-VFNS and the construction of the massive hard scattering cross sections can be found in Refs. [9, 10] and 

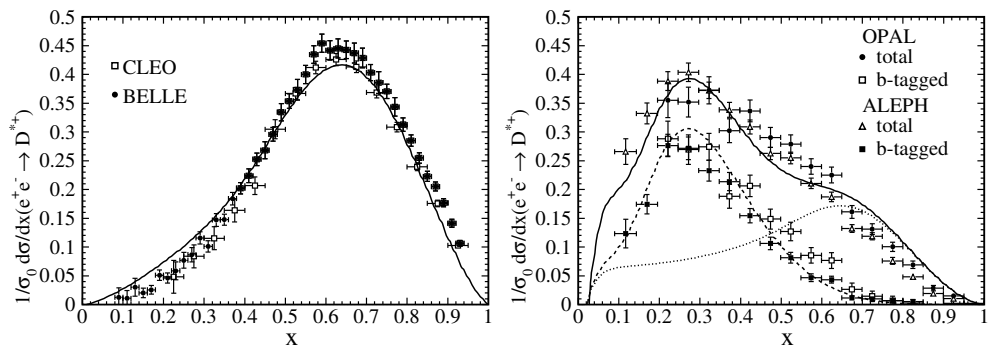

Figure 1: The cross section for inclusive $D^{* \pm}$ production in $e^{+} e^{-}$annihilation evaluated in NLO is compared with from CLEO [23] and BELLE [24] (left) as well as from the ALEPH [18] and OPAL [19] data (right). The three curves in the right figure correspond to the $Z \rightarrow c \bar{c}, Z \rightarrow b \bar{b}$ and full samples.

the conference proceedings $[13,14,15,16]$.

\section{$2.2 \quad$ Fragmentation Functions}

A crucial ingredient entering these calculation are the non-perturbative FFs for the transition of the final state parton into the observed hadron $X_{h}$. For charm-flavoured mesons, $X_{c}$, such sets of FFs have been constructed quite some time ago. For $X_{c}=D^{*+}$, FFs were extracted at $\mathrm{LO}$ and $\mathrm{NLO}$ in the $\overline{\mathrm{MS}}$ factorisation scheme with $n_{f}=5$ massless quark flavours [17] from the scaled-energy $(x)$ distribution $d \sigma / d x$ of the cross section of $e^{+} e^{-} \rightarrow D^{*+}+X$ measured by the ALEPH [18] and OPAL [19] collaborations at CERN LEP1. Recently, this analysis was extended [20] to include $X_{c}=D^{0}, D^{+}, D_{s}^{+}, \Lambda_{c}^{+}$by exploiting appropriate OPAL data [21]. In Refs. [17, 20], the starting scales $\mu_{0}$ for the DGLAP evolution of the $a \rightarrow X_{c}$ FFs in the factorisation scale $\mu_{F}^{\prime}$ have been taken to be $\mu_{0}=2 m_{c}$ for $a=g, u, \bar{u}, d, \bar{d}, s, \bar{s}, c, \bar{c}$ and $\mu_{0}=2 m_{b}$ for $a=b, \bar{b}$. The FFs for $a=g, u, \bar{u}, d, \bar{d}, s, \bar{s}$ were assumed to be zero at $\mu_{F}^{\prime}=\mu_{0}$ and were generated through the DGLAP evolution to larger values of $\mu_{F}^{\prime}$. For consistency with the $\overline{\mathrm{MS}}$ prescription for PDFs, we repeated the fits of the $X_{c}$ FFs for the choice $\mu_{0}=m_{c}, m_{b}$ [22]. This changes the $c$-quark FFs only marginally, but has an appreciable effect on the gluon FF, which is important at Tevatron energies, as was found for $D^{*+}$ production in Ref. [9]. In the meantime much more accurate data for the inclusive production of $D^{0}, D^{+}$and $D^{*+}$ mesons in $e^{+} e^{-}$annihilation have been published by the CLEO [23] and the BELLE [24] collaborations. With these data new FFs have been constructed. These fits were done in the framework of the GM-VFNS, where the finite charm- and bottom-quark masses were kept in the hard scattering cross sections. A global fit with the data from CLEO and BELLE at $10.52 \mathrm{GeV}$ together with the ALEPH [18] and OPAL [19] data at the $Z$-resonance are shown in Fig. 1 [25].

Already many years ago we made an analysis towards FFs for bottom-flavoured mesons $B^{ \pm}[26]$ by using data from the OPAL collaboration at LEP1 [27]. In the last years much more accurate measurements of the inclusive $B$ meson production at the $Z$-resonance have been done by the ALEPH [28], SLD [29] and the OPAL [30] collaborations. With the data in these references combined we have performed a new fit to obtain the FFs for $q, g, b \rightarrow B$, 


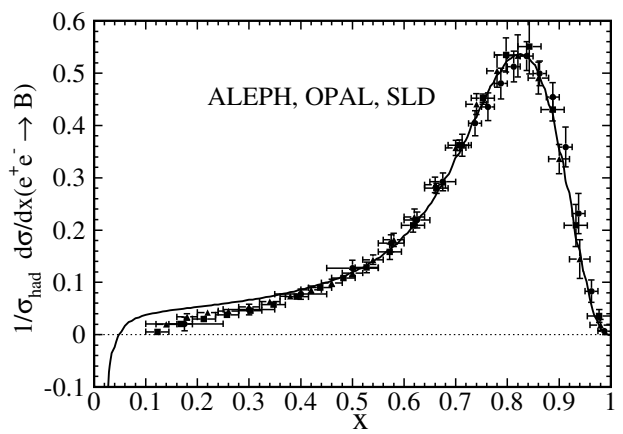

Figure 2: Comparison of the ALEPH [28] (circles), SLD [29] (triangles) and OPAL [30] (squares) data with the NLO fits using the power ansatz. The initial factorisation scale for all partons is $\mu_{0}=4.5 \mathrm{GeV}$.

where, in this case, $q$ are the light quarks including $c$. To be consistent with the starting scale of the PDFs the FFs of these light partons are assumed to vanish at the starting scale $\mu_{0}=m_{b}=4.5 \mathrm{GeV}$ and only the $b \rightarrow B \mathrm{FF}$ is parametrised by the usual power ansatz at the starting scale $\mu_{0}$. The FFs of the light quarks and the gluon are generated via DGLAP evolution at higher scales. The result of the combined fit is seen in Fig. 2. All three data sets are consistent with each other and the fit describes the data quite well in the whole $x$ range, except possibly at rather small $x[31]$.

\subsection{Input Parameters}

For the numerical results presented below we have chosen the following input. For the proton PDFs we have employed the CTEQ6.1M PDFs from the CTEQ collaboration [32, 33] and for the charmed meson fragmentation functions the sets from [22]. We have set $m_{c}=1.5 \mathrm{GeV}$, $m_{b}=5 \mathrm{GeV}$ (in the case of charmed meson production), $m_{b}=4.5 \mathrm{GeV}$ (in the case of $B$ meson production) and have used the two-loop formula for $\alpha_{s}^{\left(n_{f}\right)}\left(\mu_{R}\right)$ in the $\overline{\mathrm{MS}}$ scheme with $\alpha_{s}^{(5)}\left(m_{Z}\right)=0.118$. The theoretical predictions depend on three scales, the renormalisation scale $\mu_{R}$, and the initial- and final-state factorisation scales $\mu_{F}$ and $\mu_{F}^{\prime}$, respectively. Our default choice for hadro- and photoproduction has been $\mu_{R}=\mu_{F}=\mu_{F}^{\prime}=m_{T}$, where $m_{T}=\sqrt{p_{T}^{2}+m^{2}}$ is the transverse mass. Scale changes are controlled by $\xi_{R}$ and $\xi_{F}$, where $\xi_{R}=\mu_{R} / m_{T}, \xi_{F}=\mu_{F} / m_{T}$ and $\xi_{F}^{\prime}=\mu_{F}^{\prime} / m_{T}$.

\section{Hadroproduction}

A few years ago the CDF collaboration has published first cross section data for the inclusive production of $D^{0}, D^{+}, D^{*+}$, and $D_{s}^{+}$mesons in $p \bar{p}$ collisions [34] obtained in Run II at the Tevatron at center-of-mass energies of $\sqrt{S}=1.96 \mathrm{TeV}$. The data come as distributions $d \sigma / d p_{T}$ with $y$ integrated over the range $|y| \leq 1$ and the particle and antiparticle contributions are averaged. 

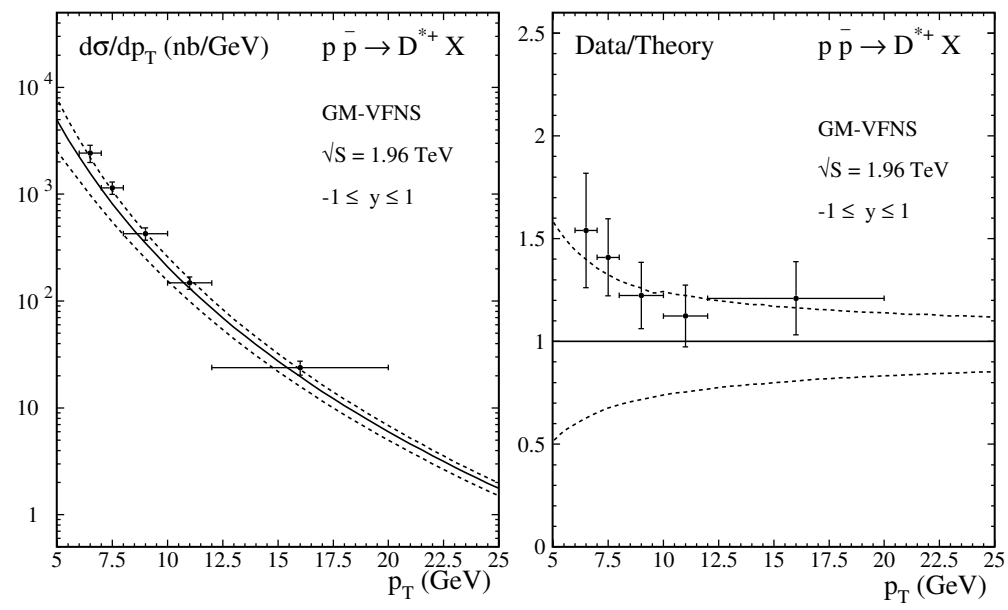

Figure 3: Comparison of the CDF data [34] with our NLO predictions for $D^{*+}$. The solid line represents our default prediction obtained with $\mu_{R}=\mu_{F}=\mu_{F}^{\prime}=m_{T}$, while the dashed lines indicate the scale uncertainty estimated by varying $\mu_{R}, \mu_{F}$, and $\mu_{F}^{\prime}$ independently within a factor of 2 up and down relative to the central values. The right figure shows the data-over-theory representation with respect to our default prediction.

Our theoretical predictions in the GM-VFNS are compared with the CDF data for $D^{\star}$ mesons on an absolute scale in Fig. 3 (left) and in the data-over-theory representation with respect to our default results in Fig. 3 (right). We find good agreement in the sense that the theoretical and experimental errors overlap, where the experimental results are gathered on the upper side of the theoretical error band, corresponding to a small value of $\mu_{R}$ and large values of $\mu_{F}$ and $\mu_{F}^{\prime}$, the $\mu_{R}$ dependence being dominant in the upper $p_{T}$ range. As is evident from Fig. 3 (right), the central data points tend to overshoot the central QCD prediction by a factor of about 1.5 at the lower end of the considered $p_{T}$ range, where the errors are largest, however. This factor is rapidly approaching unity as the value of $p_{T}$ is increased. The tendency of measurements of inclusive hadroproduction in Tevatron run II to prefer smaller renormalisation scales is familiar from single jets, which actually favour $\mu_{R}=p_{T} / 2$ [35]. It will be interesting to compare these data with predictions using the most recently constructed fragmentation functions based on the BELLE and CLEO data shown above. For more details and a comparison with the data for the $D^{0}, D^{+}$, and $D_{s}^{+}$mesons we refer to Ref. [11].

In the GM-VFNS framework we have also calculated the cross section distribution $d \sigma / d p_{T}$ of $B$-meson hadroproduction. The calculations proceed analogously to the case of $D$ mesons outlined in Ref. [9]. Now the heavy quark mass $m$ is the $b$ quark mass $m_{b}$. The $c$ quark belongs to the group of light quarks $q$, whose mass is put to zero.

The NLO cross section consists of three classes of contributions.

Class (i) contains all the partonic subprocesses with a $b, \bar{b} \rightarrow B$ transition in the final state that have only light partons $(g, q, \bar{q})$ in the initial state, the possible pairings being $g g, g q$, 


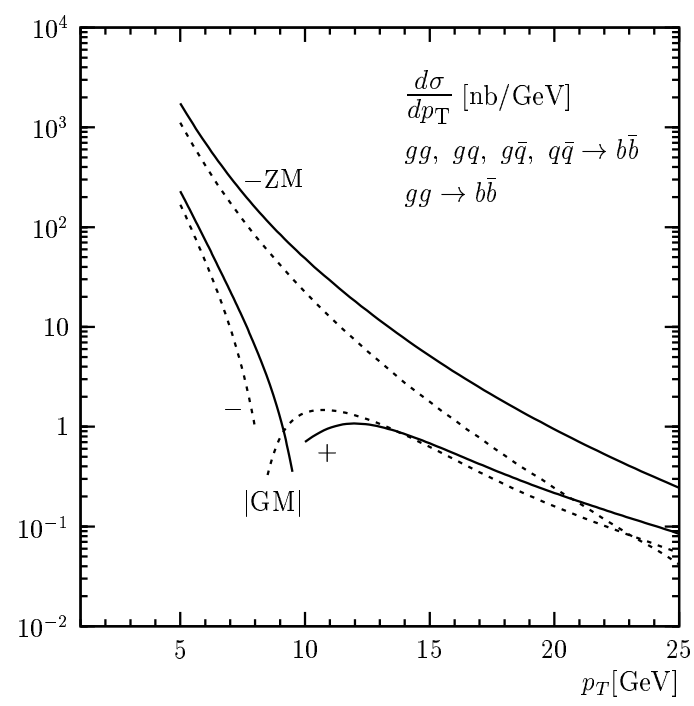

Figure 4: Transverse-momentum distribution $d \sigma / d p_{T}$ of $p \bar{p} \rightarrow B+X$ at c.m. energy $\sqrt{S}=1.96 \mathrm{TeV}$ integrated over the rapidity range $|y|<1$. The contributions of class (i) (solid lines) and their $g g$ initiated parts (dashed lines) evaluated at NLO in the ZM-VFNS (upper lines) and the GM-VFNS (lower lines) are compared.

$g \bar{q}$, and $q \bar{q}$.

Class (ii) contains all the partonic subprocesses with $b, \bar{b} \rightarrow B$ transitions in the final state that also have $b$ or $\bar{b}$ quarks in the initial state, the possible pairings being $g b, g \bar{b}, q b, q \bar{b}, \bar{q} b$, $\bar{q} \bar{b}$ and $\bar{b} \bar{b}$.

Class (iii) contains all the partonic subprocesses with a $g, q, \bar{q} \rightarrow B$ transition in the final state.

In the FFNS only the contributions of class (i) are included, but the full $m$ dependence is retained. On the other hand, in the ZM-VFNS, the contributions of all the three classes are taken into account, but they are evaluated for $m=0$. In the GM-VFNS, the class-(i) contribution of the FFNS is matched to the $\overline{\mathrm{MS}}$ scheme through appropriate subtractions of would-be collinear singularities, and is then combined with the class-(ii) and class-(iii) contributions of the ZM-VFNS. Thus only the hard scattering cross sections of class (i) carry explicit $m$ dependence. Specifically, the subtractions affect initial states involving $g \rightarrow b \bar{b}$ splittings and final states involving $g \rightarrow b \bar{b}, b \rightarrow g b$ and $\bar{b} \rightarrow g \bar{b}$ splittings, and they introduce logarithmic dependences on the initial- and final-state factorisation scales in the hard-scattering cross sections of class (i), which are compensated through NLO by the respective factorisation scale dependences by the $b$-quark PDF and the $b \rightarrow B \mathrm{FF}$, respectively. It turns out that the $q$-quark fragmentation contribution is negligible. However, the gluon fragmentation reaches approximately $50 \%$ at small values of $p_{T}$, and somewhat less towards larger values of $p_{T}$.

The explicit contributions to the hard scattering cross sections of class (i) as they contribute to the final result, after all the subtractions are made, are shown in Fig. 4. The results for $m=0$ and finite $m$ are shown in this figure as the upper and lower solid lines, 


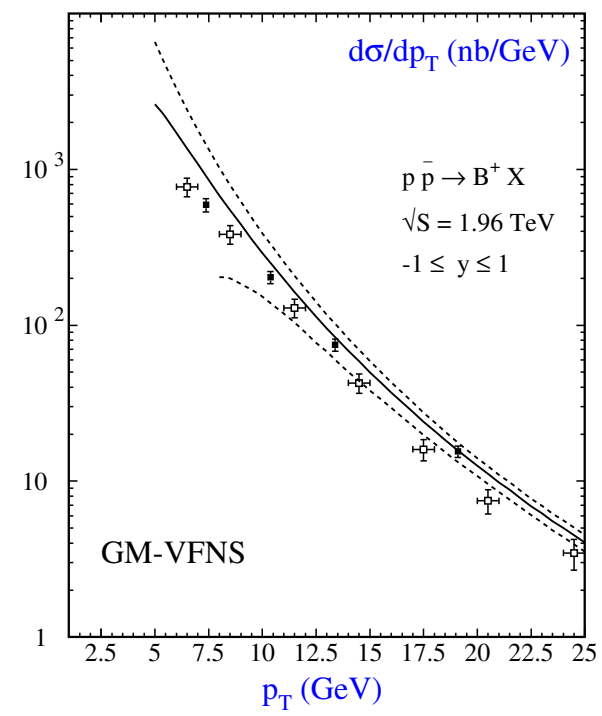

Figure 5: Transverse-momentum distribution $d \sigma / d p_{T}$ of $p \bar{p} \rightarrow B+X$ at c.m. energy $\sqrt{S}=1.96 \mathrm{TeV}$ integrated over the rapidity range $|y|<1$. The central NLO prediction with $\xi_{R}=\xi_{F}=1$ (solid line) of the GM-VFNS is compared with CDF data from Refs. [36] (open squares) and [37] (solid squares). The maximum and minimum values obtained by independently varying $\xi_{R}$ and $\xi_{F}$ in the range $1 / 2 \leq \xi_{R}, \xi_{F} \leq 2$ with the constraint that $1 / 2 \leq \xi_{R} / \xi_{F} \leq 2$ are also indicated (dashed lines).

respectively. They constitute parts of the final ZM-VFNS and GM-VFNS results. In both cases, the contributions of classes (ii) and (iii) for $m=0$ still must be added to obtain the full predictions to be compared with experimental data. The class-(i) contributions in the ZM-VFNS and GM-VFNS schemes are, therefore, entitled to be negative and they indeed are, for $p_{T} \lesssim 76 \mathrm{GeV}$ and $p_{T} \lesssim 10 \mathrm{GeV}$, respectively, as may be seen from Fig. 4. Comparing the ZM-VFNS and GM-VFNS results, we notice that the finite- $m$ effects are significant for $p_{T} \lesssim 10 \mathrm{GeV}$ and even cause a sign change for $10 \mathrm{GeV} \lesssim p_{T} \lesssim 76 \mathrm{GeV}$. However, as will become apparent below, the contributions of class (i) are overwhelmed by those of classes (ii) and (iii), so that the finite- $m$ effects are washed out in the final predictions, except for very small values of $p_{T}$. It is instructive to study the relative importance of the $g g$-initiated contributions. They are also included in Fig. 4 for $m=0$ and finite $m$ as the upper and lower dashed lines, respectively. They exhibit a similar pattern as the full class-(i) contributions and dominate the latter in the small- $p_{T}$ range. Comparing Fig. 4 with Fig. 2(c) in Ref. [9], we observe that the relative influence of the finite- $m$ effects is much smaller in the $c$-quark case, as expected because the $c$ quark is much lighter than the $b$ quark. One can also see from Fig. 4 that the difference of the class (i) contributions in the GM-VFNS and ZM-VFNS decrease with increasing $p_{T}$.

In Fig. 5 we show the comparison of the final prediction, in which all contributions of classes (i), (ii) and (iii) are combined [31] with recent Tevatron data. We compare our 
prediction to the more recent CDF data from run II in Refs. [36] (open squares) and [37] (solid squares). In this figure the solid line presents the central prediction for $\xi_{R}=\xi_{F}=1$ and the dashed lines indicate the maximum and minimum values obtained by independently varying $\xi_{R}$ and $\xi_{F}=\xi_{F}^{\prime}$ in the range $1 / 2 \leq \xi_{R}, \xi_{F} \leq 2$ with the constraint $1 / 2 \leq \xi_{R} / \xi_{F} \leq 2$. The maximum and minimum values correspond to $\xi_{F}=2$ and $\xi_{F}=1 / 2$, respectively. The variation with $\xi_{R}$ is milder than the one with $\xi_{F}$. For $\xi_{F}<1, \mu_{F}$ reaches the starting scale $\mu_{0}=m$ for the DGLAP evolution of the FFs and the $b$-quark PDF at $p_{T}=m_{b} \sqrt{1 / \xi_{F}^{2}-1}$. For smaller values of $p_{T}$, there is no prediction because the FFs and the $b$-quark PDF are put to zero for $\mu_{F}<\mu_{0}$. This explains why the $p_{T}$ distribution for $\xi_{F}=1 / 2$ only starts at $p_{T}=\sqrt{3} m_{b} \approx 7.8 \mathrm{GeV}$. The most recent data [37] nicely agree with the GM-VFNS result. They lie close to the central prediction, with a tendency to fall below it in the lower $p_{T}$ range, and they are comfortably contained within the theoretical error band. We conclude from this, that the notorious Tevatron $B$-meson anomaly with data-to-theory ratios of typically $2-3$, that has been in the literature for more than a decade, is actually not present thanks to both experimental and theoretical progress. The previous CDF data [36] based on the measurement of $J / \psi+X$ final states are compatible with the latest ones for $p_{T}<12 \mathrm{GeV}$, but are systematically below them for the larger values of $p_{T}$. This inconsistency becomes even more apparent by noticing that Fig. 4 only contains 4 out of the 13 data points for $p_{T}>12 \mathrm{GeV}$ quoted in Ref. [36] and that the omitted data points line up with the selected ones. This suggests that the systematical errors in Ref. [36] and perhaps also in ref. [37], might be underestimated and that the overall normalisation might need some adjustment.

The measured $p_{T}$ distributions of Ref. [36] reaches down to almost $p_{T}=0$ and exhibits a maximum at $p_{T} \approx 2.5 \mathrm{GeV}$. This small- $p_{T}$ behaviour is correctly reproduced in the FFNS without DGLAP-evolved FFs, which receive only contributions of class (i) without any subtractions. It is clear that our present implementation of the GM-VFNS is not suitable for cross sections in the small- $p_{T}$ region. Although the GM-VFNS is designed to approach the FFNS in its region of validity without introducing additional matching factors, to implement this numerically is not easy due to necessary cancellations between different terms in the calculation. The problem to achieve such cancellations is complicated by the extra factorisation scale; to obtain a smooth transition from the GM-VFNS to the FFNS, one has to carefully match terms that are taken into account at fixed order with terms that are resummed to higher orders in the PDFs and FFs. In addition, it remains to be investigated whether a proper scale choice in the small- $p_{T}$ range is required and helpful to ensure that the FFs and $b$-quark PDF are sufficiently suppressed already at $p_{T}=\mathcal{O}(m)$.

We extend our numerical analysis to include the NLO prediction in the FFNS with $n_{f}=4$ massless quark flavours in the initial state, which allows us to also compare with the small- $p_{T}$ data from Ref. [36]. We evaluate $\alpha_{s}^{\left(n_{f}\right)}\left(\mu_{R}\right)$ with $n_{f}=4$ and $\Lambda^{(4)}=326 \mathrm{MeV}$ [32], while we continue using the CTEQ6.1M proton PDFs [32], in want of a rigorous FFNS set with $n_{f}=4$. In the FFNS, there is no room for DGLAP-evolved FFs, and only $b, \bar{b} \rightarrow B$ transitions are included. For simplicity, we identify $b$ (anti)quarks with $B$ mesons and account for nonperturbative effects by including the branching fraction $B(b \rightarrow B)=39.8 \%$ [38] as an overall normalisation factor, i.e. we use a $b \rightarrow B$ FF of the form $D(x)=B(b \rightarrow B) \delta(1-x)$, while the $g, q, \bar{q} \rightarrow B$ FFs are put to zero. In Fig. 6, the central FFNS (dot-dashed line), ZM-VFNS (dashed line), and GM-VFNS (solid line) predictions, for $\xi_{R}=\xi_{F}=1$, are compared with the CDF data from Refs. [36, 37]. As in Fig. 4, some of the data points with $p_{T}>7 \mathrm{GeV}$ from Ref. [36] are omitted for clarity. Since the ZM-VFNS and our present 


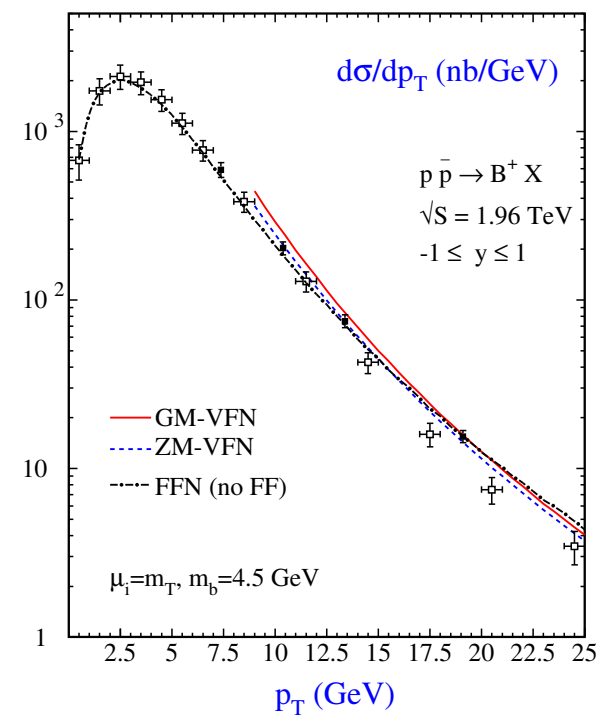

Figure 6: Transverse-momentum distribution $d \sigma / d p_{T}$ of $p \bar{p} \rightarrow B+X$ at c.m. energy $\sqrt{S}=1.96 \mathrm{TeV}$ integrated over the rapidity range $|y|<1$. The central NLO predictions in the FFNS with $n_{f}=4$ and without FFs (dot-dashed line), the ZM-VFNS (dashed line), and the GM-VFNS (solid line) are compared with CDF data from Refs. [36] (open squares) and [37] (solid squares).

implementation of the GM-VFNS are not applicable to the small- $p_{T}$ range, we show the respective predictions only for $p_{T}>2 m=9 \mathrm{GeV}$. The GM-VFNS prediction shown in Fig. 6 is identical with the central one in Fig. 5. By construction, it merges with the ZM-VFNS prediction with increasing value of $p_{T}$. In accordance with the expectation expressed in the discussion of Fig. 4, the difference between the GM-VFNS and ZM-VFNS results is rather modest also at $p_{T} \gtrsim 2 m$, since the $m$-dependent contribution, of class (i), is numerically small and overwhelmed by the $m$-independent ones, of classes (ii) and (iii). The FFNS prediction faithfully describes the peak structure exhibited by the next-to-latest CDF data [36] in the small- $p_{T}$ range and it also nicely agrees with the latest CDF data [37] way out to the largest $p_{T}$ values. In fact, for $p_{T}>4 m$, where its perturbative stability is jeopardised by unresummed logarithms of the form $\ln \left(m_{T}^{2} / m^{2}\right) \gtrsim 3$, the FFNS prediction almost coincides with the GM-VFNS one, where such large logarithms are resummed. This might be a pure coincidence, which becomes even more apparent if we also recall that the implementation of the $b, \bar{b} \rightarrow B$ transition in the FFNS is not based on a factorisation theorem and quite inappropriate for such large values of $p_{T}$.

\section{Photoproduction}

Inclusive photoproduction of $D^{\star}$ mesons, $\gamma+p \rightarrow D^{\star}+X$, has been studied in Ref. [8] where the direct part has been computed in the GM-VFNS whereas the resolved part has been included in the ZM-VFNS. In this analysis the FFs of Ref. [17] and, for the resolved 
$\gamma \mathrm{p}$ : inclusive $\mathrm{D}^{*}$

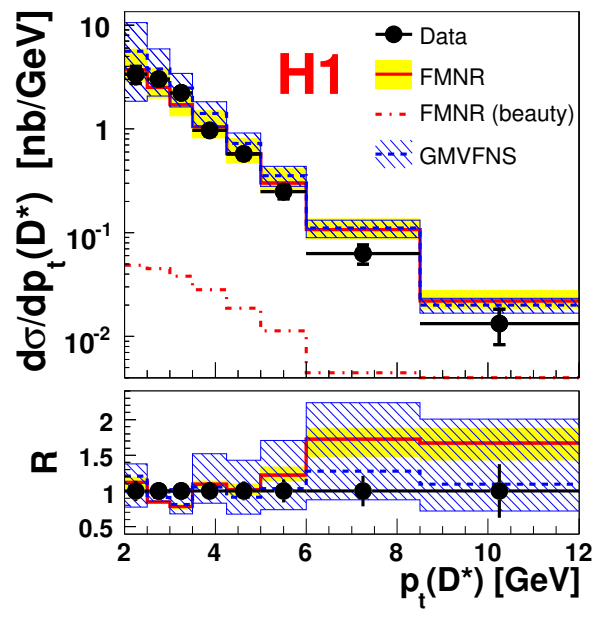

$\gamma \mathrm{p}$ : inclusive $\mathrm{D}^{*}$

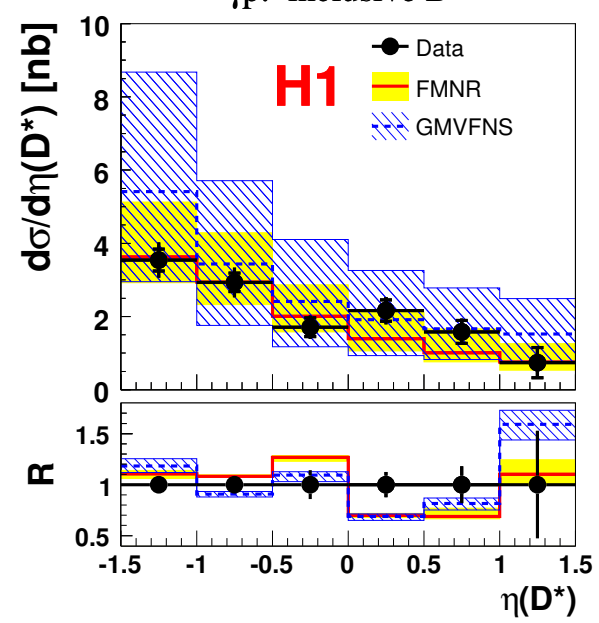

Figure 7: Inclusive $D^{*}$ cross sections as a function of $p_{t}\left(D^{*}\right)$ (left) and $\eta\left(D^{*}\right)$ (right) compared to NLO QCD calculations of FMNR [43] in the FFNS and GM-VFNS for photoproduction in the laboratory frame. The FMNR bottom contribution is shown separately for the $p_{t}\left(D^{*}\right)$ distribution.

contribution, the GRV92 photon PDFs [39] have been utilized. The other parameters have been chosen as specified in Sec. 2.3. In Fig. 6 of Ref. [8], the central numerical predictions for the $p_{T}$ distributions of the $D^{\star}$ meson have been compared with preliminary ZEUS data [40]. There exist similar data by the H1 collaboration [41] which have not been used in this analysis. As can be seen in this figure, the agreement of the $p_{T}$-distributions with the data is quite good down to $p_{T} \simeq 2 m_{c}$ and the mass effects turn out to be small. In order to extend the range of applicability of the GM-VFNS into the region $p_{T}<3 \mathrm{GeV}$ more work on the matching to the 3-fixed flavour theory would be needed. Figs. $7-9$ of Ref. [8], showing results for the rapidity $(y)$, invariant mass $(W)$ and inelasticity $\left(z\left(D^{\star}\right)\right)$ distributions, have to be taken with a grain of salt since they receive large contributions from the transverse momentum region $1.9<p_{T}<3 \mathrm{GeV}$ which is outside the range of validity of the present theory. With the work in Ref. [9], it was possible to include also the resolved part in the GM-VFNS. This has been done and the predictions in the complete GM-VFNS framework at NLO, combined with updated FFs [22], have been compared with recent H1 photoproduction data [42]. The results of the calculation and the comparison with the data is shown in Fig. 7. In this figure also the predictions in the FFNS based on the FMNR program [43] are shown. The experimental cross section as a function of $p_{T}$ falls steeply with increasing $p_{T}$ as predicted by both calculations. FMNR predicts a distribution which decreases less steeply at large $p_{T}$ than the data as is seen more clearly from the plot of the ratio of the theoretical over the measured cross section. Also in Fig. 7 the differential cross section as a function of the pseudorapidity $\eta\left(D^{*}\right)$ is shown. This cross section decreases with increasing $\eta$. Both calculations predict a similar shape and agree nicely with the data. The GM-VFNS prediction shows a larger scale dependence. Otherwise the two calculations 
give rather similar results, which is remarkable, considering the very different ingredients of the two approaches.

\section{Summary}

We have discussed one-particle inclusive production of heavy-flavoured hadrons in hadronhadron and photon-proton collisions in a massive variable-flavour-number scheme (GMVFNS). The importance of a unified treatment of all these processes, based on QCD factorisation theorems, has been emphasised, in order to provide meaningful tests of the universality of the FFs and hence of QCD. At the same time, it is necessary to incorporate heavy-quark mass effects in the formalism since many of the present experimental data points lie in a kinematical region where the hard scale of the process is not much larger than the heavyquark mass. This is achieved in the GM-VFNS, which includes heavy-quark mass effects and still relies on QCD factorization. We have discussed numerical results for two reactions. In general, the description of the transverse momentum spectra is quite good down to transverse momenta $p_{T} \simeq 2 m$. Extending the range of applicability of our scheme to smaller $p_{T}$ would require more work on the matching to the corresponding theories in the fixed flavor number scheme.

\section{Acknowledgments}

The author would like to thank the organisers of the DIS 2007 workshop for the kind invitation, B. A. Kniehl, I. Schienbein and H. Spiesberger for their collaboration.

\section{References}

[1] Slides: http://indico.cern. ch/contributionDisplay.py? contribId=10\&sessionId=2\&conf Id=9499

[2] J. C. Collins, D. E. Soper, and G. Sterman, in Perturbative Quantum Chromodynamics, edited by A. H. Mueller (World Scientific, 1989).

[3] J. C. Collins and D. E. Soper, Ann. Rev. Nucl. Part. Sci. 37, 383 (1987).

[4] P. Nason, S. Dawson, and R.K. Ellis, Nucl. Phys. B303, 607 (1988); B327, 49 (1989); B335, 260(E) (1989); W. Beenakker, H. Kuijf, W.L. van Neerven, and J. Smith, Phys. Rev. D40, 54 (1989); W. Beenakker, W.L. van Neerven, R. Meng, G.A. Schuler, and J. Smith, Nucl. Phys. B351, 507 (1991); I. Bojak and M. Stratmann, Phys. Rev. D67, 034010 (2003).

[5] M. Cacciari, M. Greco and P. Nason, JHEP 05007 (1998); M. Cacciari and P. Nason, Phys. Rev. Lett. 89122003 (2002).

[6] G. Kramer and H. Spiesberger, Eur. Phys. J. C22, 289 (2001).

[7] G. Kramer and H. Spiesberger, Eur. Phys. J. C28, 495 (2003).

[8] G. Kramer and H. Spiesberger, Eur. Phys. J. C38, 309 (2004).

[9] B. A. Kniehl, G. Kramer, I. Schienbein, and H. Spiesberger, Phys. Rev. D71, 014018 (2005).

[10] B. A. Kniehl, G. Kramer, I. Schienbein, and H. Spiesberger, Eur. Phys. J. C41, 199 (2005).

[11] B. A. Kniehl, G. Kramer, I. Schienbein, and H. Spiesberger, Phys. Rev. Lett. 96, 012001 (2006).

[12] J. C. Collins, Phys. Rev. D58, 094002 (1998).

[13] I. Schienbein, Open heavy-flavour photoproduction at NLO, Proceedings of the Ringberg Workshop, New Trends in HERA Physics 2003, edited by G. Grindhammer, B. A. Kniehl, G. Kramer and W. Ochs, World Scientific, 2004, p. 197;

[14] I. Schienbein, Proceedings of the 12th International Workshop on Deep Inelastic Scattering (DIS 2004), Strbske Pleso, Slovakia, 14-18 Apr 2004, hep-ph/0408036; 
[15] B. A. Kniehl, G. Kramer, I. Schienbein, and H. Spiesberger, Proceedings of the 13th International Workshop on Deep Inelastic Scattering (DIS 2005), Madison, Wisconsin, USA, April 27 - May 1,2005, hep-ph/0507068.

[16] J. Baines et al, Summary report of the heavy-flavour working group of the HERA-LHC Workshop, hep-ph/0601164.

[17] J. Binnewies, B. A. Kniehl, and G. Kramer, Phys. Rev. D58, 014014 (1998).

[18] R. Barate et al., [ALEPH Collaboration], Eur. Phys. J. C16, 597 (2000).

[19] K. Ackerstaff et al., [OPAL Collaboration], Eur. Phys. J. C1, 439 (1998).

[20] B. A. Kniehl and G. Kramer, Phys. Rev. D71, 094013 (2005).

[21] G. Alexander et al., [OPAL Collaboration], Z. Phys. C72, 1 (1996).

[22] B. A. Kniehl and G. Kramer, Phys. Rev. D74 037502 (2006).

[23] M. Artuso et al. [CLEO Collaboration], Phys. Rev. D70 112001 (2004)

[24] R. Seuster et al. [Belle Collaboration], Phys. Rev. D73 032002 (2006)

[25] T. Kneesch, B. A. Kniehl, G. Kramer and I. Schienbein, in preparation

[26] J. Binnewies, B. A. Kniehl and G. Kramer, Phys. Rev. D58 034016 (1998)

[27] G. Alexander et al. [OPAL Collaboration], Phys. Lett. B364 93 (1995).

[28] A. Heister et al. [ALEPH Collaboration], Phys. Lett. B512 30 (2001).

[29] K. Abe et al. [SLD Collaboration], Phys. Rev. D65 092006 (2002), [Erratum-ibid. D66 079905 (2002)].

[30] G. Abbiendi et al. [OPAL Collaboration], Eur. Phys. J. C29 463 (2003).

[31] B. A. Kniehl, G. Kramer, I. Schienbein, and H. Spiesberger, arXiv:0705.4392 [hep-ph] (2007).

[32] J. Pumplin et al., JHEP 07, 012 (2002)

[33] D. Stump et al., JHEP 10, 046 (2003).

[34] D. Acosta et al., [CDF Collaboration], Phys. Rev. Lett. 91, 241804 (2003).

[35] R. Field, for the CDF Collaboration, in Proceedings of the XIII ${ }^{\text {th }}$ International Workshop on Deep Inelastic Scattering (DIS05), Madison, Wisconsin, 2005 (American Institute of Physics, Melville, to be published); B. Davies, for the D0 Collaboration, ibid.

[36] D. Acosta et al. [CDF Collaboration], Phys. Rev. D71 032001 (2005).

[37] A. Abulencia et al. [CDF Collaboration], Phys. Rev. D75 012010 (2007).

[38] W.M. Yao et al. (Particle Data Group), J. Phys. G33, 1 (2006).

[39] M. Glück, E. Reya, and A. Vogt, Phys. Rev. D46, 1973 (1992).

[40] ZEUS Collaboration, S. Chekanov et al., 31st International Conference on High Energy Physics, ICHEP02, July 24-31 2002, Amsterdam, Abstract 786; see also: ZEUS Collaboration, http://www-zeus.de/public-plots, Measurement of $D^{\star}$ photoproduction at HERA, 2003.

[41] H1 Collaboration, International Europhysics Conference on High Energy Physics, EPS03, July 17-23 2003, Aachen, Abstract 097, DESY-H1prelim-03-071 and earlier H1 papers given in this reference.

[42] A. Aktas et al. [H1 Collaboration], Eur. Phys. J. C50 251 (2007).

[43] S. Frixione, P. Nason and G. Ridolfi, Nucl. Phys. B454 3 (1995); S. Frixione, M. L. Mangano, P. Nason and G. Ridolfi, Phys. Lett. B348 633 (1995). 